\title{
Perfil Antropométrico de Remeros Juveniles Chilenos
}

\author{
Anthropometric Profile of Chilean Junior Rowers \\ Rubén Gajardo Burgos*; Cristian Gómez Coronado**; Jorge Flández Valderrama***; \\ Sergio Martínez Huenchullán* \& Manuel Monrroy Uarac*
}

GAJARDO, B. R.; GÓMEZ, C. C.; FLÁNDEZ, V. J.; MARTÍNEZ, H. S. \& MONRROY, U. M. Perfil antropométrico de remeros juveniles chilenos. Int. J. Morphol., 31(3):797-801, 2013.

RESUMEN: El objetivo de esta investigación fue describir las características antropométricas de remeros varones de la categoría juvenil chilenos que han participado en un proceso de selección durante los años 2011 y 2012. Se evaluaron 27 deportistas con una

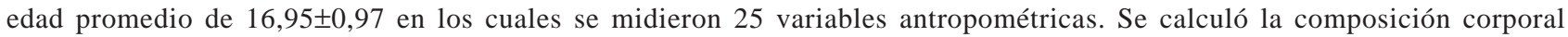
pentacompartimental, el somatotipo y la proporcionalidad. Se utilizó estadística descriptiva para la caracterización de los deportistas. El promedio de los resultados mostró que los remeros juveniles chilenos presentan un porcentaje de masa muscular elevado, de tejido adiposo bajo y un somatotipo mesomórfico balanceado.

PALABRAS CLAVE: Antropometría; Somatotipo; Composición corporal; Remo.

\section{INTRODUCCIÓN}

La valoración antropométrica es una de las herramientas más utilizadas en la caracterización de deportistas de distintas disciplinas y categorías debido a su accesibilidad y costos. Se ha demostrado que durante la etapa de especialización deportiva, los deportistas adquieren algunas características antropométricas diferenciales, en las cuales influyen factores sociales, hereditarios, nutritivos y de entrenamiento, entre otros (Alacid et al., 2011).

Una de las principales funciones de esta valoración es el control del entrenamiento y de la evolución de estos programas a través del tiempo (Lozovina \& Pavicic, 2004). Otro punto destacado es la utilización de esta herramienta en el proceso de selección de talentos deportivos (FonsecaToledo et al., 2010), ya que las dimensiones antropométricas muchas veces juegan un rol fundamental en el rendimiento deportivo (Norton \& Olds, 1996; Alacid et al.). Por esto se hace esencial obtener valores normativos de sujetos con un nivel elevado de rendimiento para obtener un modelo antropométrico propio.

Los remeros han sido caracterizados en un número importante de estudios, entre los cuales se destacan algunos en población juvenil (Bourgois et al., 2000). En estos se enfatiza que el remo es un deporte en donde se destacan las aptitudes de fuerza y resistencia, dos aptitudes físicas relacionadas con la composición corporal. Rusell et al. (1998) destaca la importancia de la masa y tamaño corporal en el rendimiento deportivo. Además, en esta especialidad, las características antropométricas están ineludiblemente relacionadas con el rendimiento y la formación de las distintas tripulaciones.

El objetivo de esta investigación fue describir las características antropométricas, composición corporal, somatotipo y proporcionalidad de los remeros de la categoría juvenil chilenos con el fin de obtener valores referenciales para futuros procesos de selección y evaluación de los programas de entrenamiento.

\section{MATERIAL Y MÉTODO}

Se evaluaron a 27 remeros varones entre los 15 y 18 años los cuales participaron de los procesos de Selección Nacional entre los años 2011 y 2012 organizados por la Federación Chilena de Remo. Sus horas de entrenamiento semanal es entre 15 y 20 horas.

* Escuela de Kinesiología, Universidad Austral de Chile, Valdivia, Chile.

** Federación Chilena de Remo, Chile.

**** Escuela de Pedagogía en Educación Física, Deporte y Recreación, Universidad Austral de Chile, Valdivia, Chile. 
Las variables que se midieron fueron las que componen el perfil restringido propuesto por la International Society for the Advancement of Kineanthropometry (ISAK) (Marfell-Jones et al., 2006). Estas medidas fueron tomadas por un antropometrista con acreditación de nivel II ISAK. Las medidas se tomaron dos veces; si la diferencia entre estas era superior a $5 \%$ en los pliegues o $1 \%$ en el resto de las variables se procedía a realizar una tercera medición. Para el análisis posterior se utilizo la media, en el caso de utilizar 2 medidas o la mediana si se utilizaron 3 mediciones. El registro se realizó en una en hoja de cálculo Excel para determinar de forma inmediata el porcentaje de error.

Para la determinación del peso se utilizó una balanza MEDISANA PST modelo 40420, la cual posee $100 \mathrm{~g}$ de precisión y una capacidad máxima de 180 kilogramos. Para el resto de las variables se utilizó un kit de antropometría Rosscraft S.R.L. de fabricación MERCOSUR, el cual posee un cáliper Gaucho Pro de 0,5 $\mathrm{mm}$ de precisión, antropómetro grande con $0,1 \mathrm{~mm}$ de precisión, antropómetro pequeño con $0,01 \mathrm{~mm}$ de precisión, una huincha métrica metálica de $1 \mathrm{~mm}$ de precisión y un segmómetro con $1 \mathrm{~mm}$ de precisión. Para la talla sentado se utilizó un cajón de madera de $40 \mathrm{~cm}$.

Se caracterizó a los deportistas a través del somatotipo según el modelo de Carter \& Heath (1990). Para la determinación de la composición corporal se utilizó el modelo pentacompartimental descrito por Kerr (1988). Se realizó el cálculo de los valores $\mathrm{Z}$ según el modelo de proporcionalidad del Phantom de Ross \& Marfell-Jones (1991) y para el índice esquel se dividió la talla sentado por la talla corporal.

Se realizó un análisis estadístico descriptivo, utilizando medidas de tendencia como la media y de dispersión como la desviación estándar, además de los valores mínimos y máximos.

\section{RESULTADOS Y DISCUSIÓN}

Los valores de las medias y su respectiva desviación estándar, mínimo y máximo de las variables evaluadas se muestran en la Tabla I. Los deportistas presentaron un Índice de masa corporal (IMC) de $25,50 \pm 1,88 \mathrm{Kg} / \mathrm{m}^{2}$, lo cual se categoriza como sobrepeso para la World Health Organization (WHO) y una mayor longitud de la envergadura, que es la distancia entre los puntos dedales de la mano derecha y de la mano izquierda cuando los miembros superiores están en abducción de $90^{\circ}$ grados (Sirvent \& Garrido, $2009)$, en relación a la talla corporal $(183,19 \pm 6,75$ vs $177,11 \pm 5,30)$.
El somatotipo de esta población, el cual establece diferencias en la forma y refleja adiposidad, robustez y linealidad relativa, se categorizó como mesomórfico balanceado $(2,62-4,75-2,41)$, lo cual indica un alto desarrollo musculoesquelético, con diámetros óseos grandes, músculos de gran volumen, poca grasa subcutánea y gran volumen por unidad de altura (Norton \& Olds) (Tabla II). Estos valores son similares a los encontrados por Rodriguez et al., (2010) en donde determinaron el somatotipo en población físicamente activa chilena.

En relación a la composición corporal se encontró un elevado porcentaje de masa muscular y un bajo porcentaje de tejido adiposo. A conocimiento de los autores existen pocas investigaciones en remeros juveniles y ninguna en donde se utilice el método pentacompartimental (compartimientos adiposo, muscular, residual, óseo y piel) en este tipo de población. Es sabido que el tejido adiposo disminuye la capacidad de desarrollar algunas capacidades como la fuerza explosiva (Davis et al., 2003) y que porcentajes elevados de masa corporal mejoran el rendimiento deportivo de los remeros (Slater et al., 2005) (Tabla III). Debido a que los deportistas evaluados en esta investigación son considerados como seleccionables para la Federación Chilena de Remo, sus valores antropométricos pueden ser tomados como de referencia a nivel local.

La clasificación por peso de los remeros se realiza desde el los Campeonatos Mundiales de 1974 y los Juegos Olímpicos de 1996 para las categorías adultas, pero no para los juveniles. Un remero de categoría ligera debe pesar menos de $72,5 \mathrm{~kg}$ para un bote single y en promedio, para un bote por equipo, menos de $70 \mathrm{~kg}$ por bote. Esto genera el problema de que debido a la talla promedio de los remeros chilenos $(177,11 \pm 5,30)$ es difícil optar a formar deportistas de categoría Open (sobre los 72,5 kg) por lo que en su etapa juvenil no pueden elevar demasiado su peso corporal. Si comparamos nuestros resultados con los finalistas de Campeonatos Mundiales de la categoría, nuestros deportistas se encuentran en desventaja en cuanto a peso y talla corporal, dos variables que aparecen como factores importantes en el rendimiento en esta categoría (Bourgois et al.).

\section{CONCLUSIÓN}

Los deportistas mostraron un porcentaje de masa muscular elevado, de tejido adiposo bajo y un somatotipo mesomórfico balanceado. Este artículo aporta la descripción de los remeros juveniles seleccionados chilenos, la cual puede ser utilizada tanto para procesos de selección de talentos en esta disciplina como para la planificación del entrenamiento. 
Tabla I. Valoración de las variables antropométricas de remeros juveniles chilenos.

\begin{tabular}{lcccc}
\hline JE & Media & DE & Máxima & Mínima \\
\hline Edad & 16,95 & 0,97 & 18,70 & 14,37 \\
Peso (kg) & 73,63 & 5,50 & 83,00 & 62,30 \\
Talla (cm) & 177,11 & 5,30 & 186,70 & 165,70 \\
ÍMC (kg/mt) & 25,50 & 1,88 & 26,98 & 19,40 \\
Talla sentado (cm) & 93,84 & 2,63 & 99,50 & 89,20 \\
Envergadura* & 183,19 & 6,75 & 194,20 & 167,10 \\
PL Tríceps (mm) & 9,73 & 2,47 & 15,60 & 5,40 \\
PL Subescapular (mm) & 8,02 & 1,64 & 12,00 & 4,50 \\
PL Supraespinal (mm) & 9,55 & 4,32 & 21,50 & 3,30 \\
PL Abdominal (mm) & 14,09 & 5,99 & 26,75 & 4,60 \\
PL Muslo frontal (mm) & 12,34 & 3,32 & 19,50 & 6,00 \\
PL Pantorrilla medial (mm) & 8,35 & 2,88 & 14,75 & 3,00 \\
Suma de 6 pliegues & 62,08 & 16,37 & 94,70 & 30,60 \\
PR Brazo relajado (cm) & 29,63 & 1,57 & 32,65 & 27,00 \\
PR Brazo flexionado en tensión (cm) & 31,95 & 1,50 & 35,50 & 29,50 \\
PR Antebrazo máximo (cm) & 27,45 & 1,18 & 30,15 & 25,40 \\
PR Tórax mesoesternal (cm) & 95,98 & 3,87 & 104,00 & 90,00 \\
PR Cintura (cm) & 77,40 & 3,65 & 84,10 & 69,68 \\
PR Cadera (cm) & 95,93 & 3,86 & 101,60 & 85,40 \\
PR Muslo (cm) & 57,72 & 2,94 & 63,75 & 51,55 \\
PR Muslo medial (cm) & 54,11 & 2,54 & 59,95 & 49,75 \\
PR Pantorrilla (cm) & 35,98 & 1,63 & 38,50 & 32,85 \\
D Biacromial (cm) & 40,87 & 1,81 & 45,40 & 37,30 \\
D Tórax transverso (cm) & 27,98 & 1,31 & 30,15 & 25,20 \\
D Tórax antero-posterior (cm) & 19,27 & 1,14 & 22,00 & 17,55 \\
D Bi-iliocrestídeo (cm) & 28,60 & 1,24 & 30,50 & 25,65 \\
D Humeral (cm) & 7,06 & 0,33 & 7,70 & 6,34 \\
D Femoral (cm) & 9,85 & 0,43 & 11,14 & 9,21 \\
\hline PL: Plieg; PR: Pero & (cm & & &
\end{tabular}

PL: Pliegues; PR: Perímetros; D: Diámetros; DE: Desviación estandar.

Fig. 1. Somatocarta de remeros juveniles chilenos. Los círculos negros pertenecen a cada participante. El círculo vacío es el promedio.

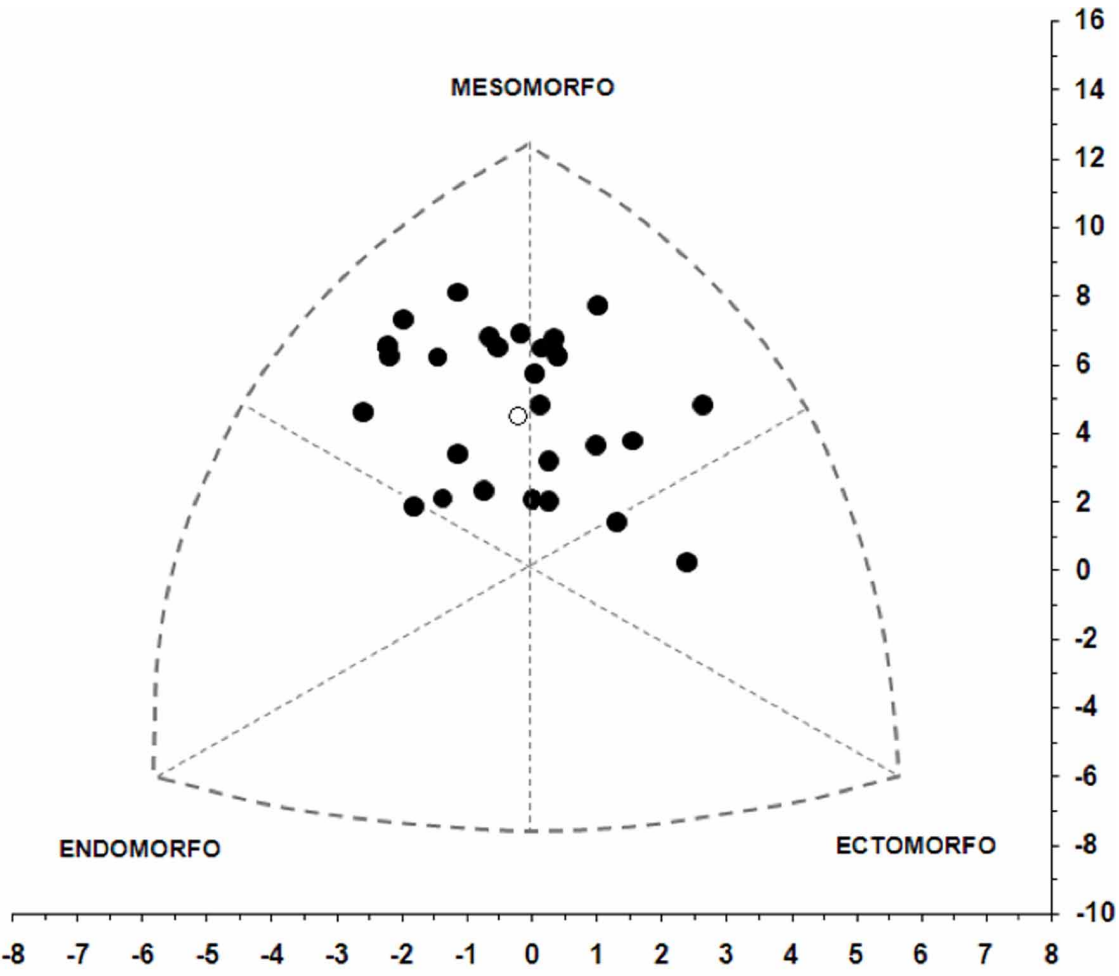


Tabla II. Somatotipo de remeros juveniles chilenos.

\begin{tabular}{lcccc}
\hline Variables & Media & DE & Máxima & Mínima \\
\hline Endomorfismo & 2,62 & 0,79 & 4,30 & 1,10 \\
Mesomorfismo & 4,75 & 0,69 & 5,8 & 3,30 \\
Ectomorfismo & 2,41 & 0,98 & 4,90 & 0,90 \\
\hline
\end{tabular}

Tabla III. Composición corporal de remeros juveniles chilenos.

\begin{tabular}{lcccc}
\hline Variables & Media & DE & Máxima & Mínima \\
\hline Masa adiposa (\%) & 24,18 & 3,60 & 31,94 & 19,21 \\
Masa muscular (\%) & 47,92 & 2,84 & 51,89 & 42,13 \\
Masa ósea (\%) & 11,81 & 0,70 & 13,04 & 10,53 \\
Masa residual (\%) & 10,82 & 0,59 & 12,12 & 9,84 \\
Masa piel (\%) & 5,23 & 0,38 & 6,41 & 4,74 \\
\hline
\end{tabular}

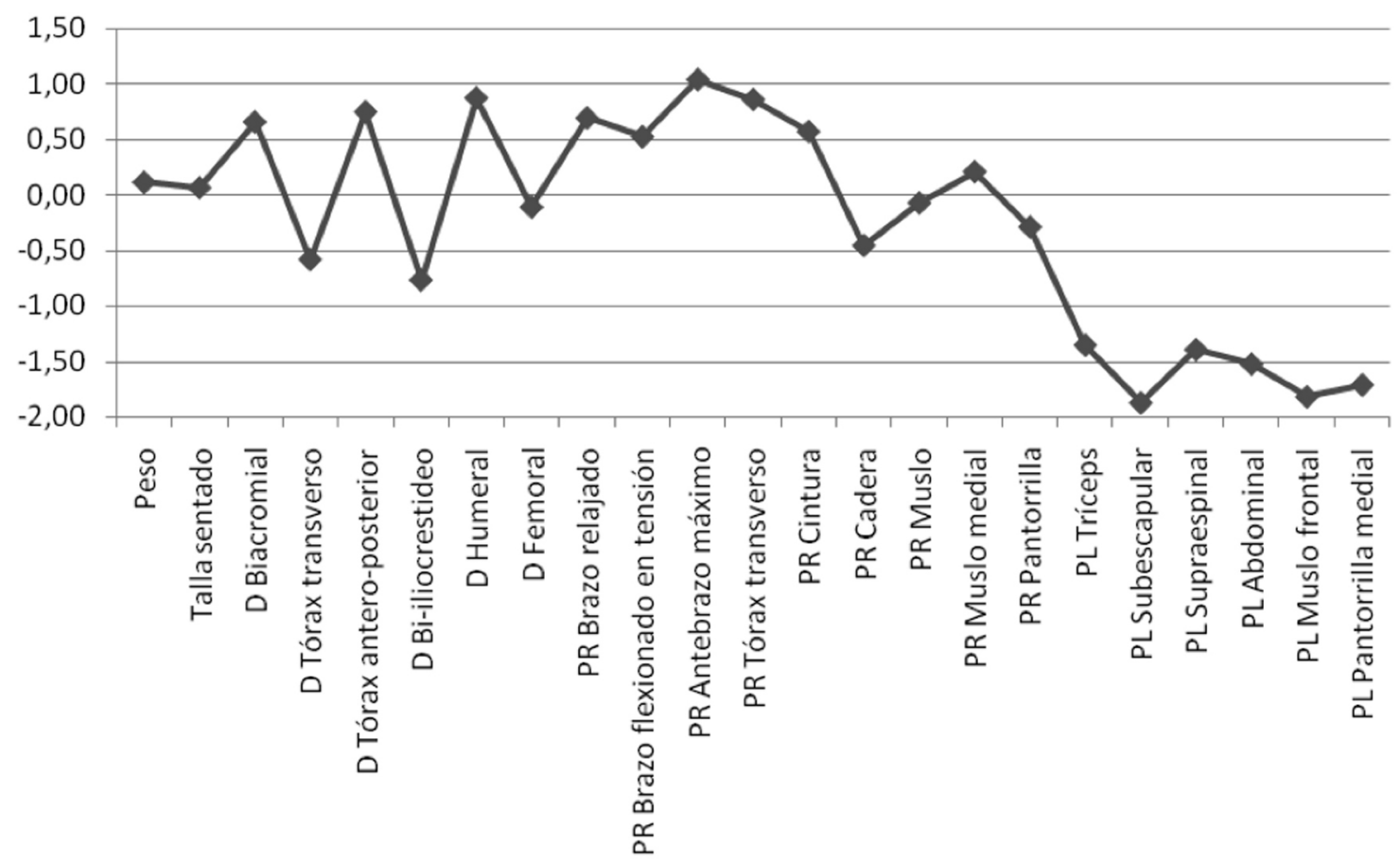

Fig. 2. Proporcionalidad (Valores Z) de remeros juveniles chilenos. PL: Pliegues; PR: Perímetros; D: Diámetros.

GAJARDO, B. R.; GÓMEZ, C. C.; FLÁNDEZ, V. J.; MARTÍNEZ, H. S. \& MONRROY, U. M. Anthropometric profile of Chilean junior rowers. Int. J. Morphol., 31(3):797-801, 2013.

SUMMARY: The aim was to describe the anthropometric characteristics of Chilean junior category male rowers who participated in a selection process during the years 2011 and 2012. We evaluated 27 athletes with a mean age of $16.95 \pm 0.97$ in which 25 anthropometric variables were measured. We calculated pentacompartimental body composition, somatotype and proportionality. Descriptive statistics were used to characterize athletes. The average of results showed that Chilean junior rowers have a high percentage of muscle mass, low body fat and a balanced mesomorphic somatotype.

KEY WORDS: Anthropometry; Somatotype; Body composition; Rower. 


\section{REFERENCIAS BIBLIOGRÁFICAS}

Alacid, F.; Muyor, J. M. \& López-Miñarro, P. A. Perfil antropométrico del canoísta joven de aguas tranquilas. Int. $J$. Morphol., 29(3):835-40, 2011.

Bourgois, J.; Claessens, A.; Vrijens, J.; Philippaerts, R.; Van Renterghem, B.; Thomis, M.; et al. Anthropometric characteristics of elite male junior rowers. Br. J. Sports Med., 34(3):213-17, 2000.

Carter, J. E. L. \& Heath, B. H. Somatotyping: development and application. Cambridge, Cambridge University Press, 1990.

Davis, S.; Briscoe, D.; Marcowski, C.; Seville, S. \& Taylor, C. Physical Characteristics that Predict Vertical Jump Performance in Recreational Male Athletes. Phys. Ther. Sport, 4(4):167-74, 2003.

Fonceca-Toledo, C.; Roquetti, P. \& Fernandes-Filho, J. Perfil antropométrico de atletas brasileiros de voleibol infanto juvenil em diferentes níveis de qualificação esportiva. Rev. Salud Pública, 12(6):915-28, 2010.

Kerr, D. A. An anthropometric method for fractionation of skin, adipose, bone, muscle and residual masses in males and females age 6 to 77 years. M.Cs. Kinesiology Tesis, Simon Fraser University, British Columbia, 1988.

Lozovina, V. \& Pavicic, L. Anthropometric Changes in Elite Male Water Polo Players: Survey in 1980 and 1995. Croat. Med. J., 45(2):202-5, 2004.

Marfell-Jones, M.; Olds, T.; Stewart, A. \& Carter, L. International standards for anthropometric assessment. Potchefstroom, South Africa, ISAK, 2006.

Norton, K. \& Olds, T. Antropométrica. Marrickville, Southwood Press, 1996.

Rodriguez, R. F. J.; Almagià, F. A. A.; Yuing, F. T.; Binvignat, G. O. \& Lizana, A. P. Composición corporal y somatotipo referencial de sujetos físicamente activos. Int. J. Morphol., 28(4):1159-65, 2010.

Ross, W. D. \& Marfell-Jones, M. Kinanthropometry. En: MacDougal, J.; Wenger, H. \& Green, H. (Eds.). Physiological testing of the high performance athlete. 2nd ed. Champaign (IL), Human Kinetics, 1991. pp.223-308.

Russell, A. P.; Le Rossignol, P. F. \& Sparrow, W. A. Prediction of elite schoolboy 2000-m rowing ergometer performance from metabolic, anthropometric and strength variables. J. Sports Sci., 16(8):749-54, 1998.

Sirvent, J. E. \& Garrido, R. P. Valoración antropométrica de la composición corporal: Cineantropometría. Valencia, Publicaciones de la Universidad de Alicante, 2009.
Slater, G.; Rice, A. J.; Mujika, I.; Hahn, A.; Sharpe, K. \& Jenkins, D. Physique traits of lightweight rowers and their relationship to competitive success. Br. J. Sports Med., 39(10):736-41, 2005.

\author{
Dirección para Correspondencia: \\ Klgo. Mg. Rubén E. Gajardo Burgos \\ Escuela de Kinesiología \\ Universidad Austral de Chile \\ Valdivia \\ CHILE
}

Tel: (063) 293727

Email: ruben_gajardo@yahoo.com

Recibido: 23-12-2012

Aceptado: 01-05-2013 